\title{
An Effective LS-SVM Based Approach for Surface Roughness Prediction in Machined Surfaces
}

\author{
Nian Zhang a and Devdas Shetty ${ }^{\mathrm{b}}$ \\ ${ }^{a}$ Department of Electrical and Computer Engineering, University of the District of Columbia, 4200 \\ Connecticut Avenue, NW, Washington, D.C. 20008 USA \\ ${ }^{\mathrm{b} S} \mathrm{School}$ of Engineering and Applied Sciences, University of the District of Columbia, 4200 Connecticut \\ Avenue, NW, Washington, D.C. 20008 USA \\ anzhang@udc.edu and ${ }^{\mathrm{b}}$ devdas.shetty@udc.edu
}

\begin{abstract}
An effective least squares support vector machine (LS-SVM) based approach was developed to predict the surface roughness in machined surface. The real AISI4340 steel and AISID2 steel data set was used to conduct the experiments. The analysis of variance (ANOVA) was used to validate the assumption of normal distribution, as well as the independent distribution of the errors. For the neural networks model, with $70 \%, 15 \%$, and $15 \%$ of data as training, validation, and testing data, the best validation error is 0.0097343 . The training error is $9.08888 \mathrm{e}-4$ and the testing error is $1.09510 \mathrm{e}-1$ accordingly. NN methods also discovered the correlation between the predicted surface roughness $(\mathrm{Ra})$ and the actual surface roughness in the form of predicted $\mathrm{Ra}$ $\sim=0.41^{*}$ Actual $\mathrm{Ra}+0.2$. The LS-SVM performance was also compared to the analysis of variance (ANOVA) method, and neural networks model trained by LevenbergMarquardt algorithm. The experimental results showed that the proposed LS-SVM algorithm produced a determination coefficient of $\mathrm{D}_{\mathrm{c}}^{2}=$ 0.9439, which is higher than the ANOVA and NN results of 0.1917 and 0.7266 .
\end{abstract}

\section{Keywords:}

Surface roughness prediction; least squares support vector machine (LS-SVM); neural networks; Levenberg-Marquardt algorithm; ANOVA; machined surfaces.

\section{Introduction}

Surface roughness is a critical quality index which determines the quality of machined surfaces and is influenced by the cutting parameters [1]. Surface roughness prediction has very important applications in manufacturing industries, environmental sciences, and military applications [2].

Many techniques have been developed to predict surface roughness. Sonar et al. [3] used radial basis neural networks for prediction of surface roughness in turning of mild steel and concluded that radial basis neural networks model are slightly inferior when compared to multilayer perceptron model. Naidu et al. [4] presented that the neural networks models are superior to regression models. Caydas and Ekici [5] developed three different types of support vector machines (SVMs) tools including least squares SVM (LS-SVM), Spider SVM and SVM-KM models to estimate the surface roughness values. The prediction results showed that the all SVMs performed better than neural networks. However, the training data are relatively intact. Wang et al [6] applied LSSVM algorithms for prediction model of surface roughness for grinding machining operation. Result shows that LS-SVM outperformed the RBF neural networks method. However, the LS-SVM needs to be compared with more efficient neural networks.

Given this context, it is imperative to develop an effective LS-SVM and compare its performance with the neural networks and 
analysis of variance methods for surface roughness prediction.

This paper is organized as follows. In Section 2, problem statement is described. In Section 3, the principle of least squares support vector machines (LS-SVM) and Neural Networks with Levenberg-Marquardt Optimization are illustrated in detail. In Section 4 , experimental results and discussions are demonstrated. In Section 5, the comparison of ANOVA, NN, and LS-SVM on the surface roughness prediction was presented. In Section 6 , the conclusions are given.

\section{Problem statement}

Given the sample of $\mathrm{N}$ points $\left\{x_{i}, y_{i}\right\}_{i=1}^{N}$, with input vectors $x_{i} \in \mathbb{R}^{p}$ and output values $y_{i} \in \mathbb{R}$, the goal is to estimate a model of the form:

$y_{i}=w^{T} \emptyset\left(x_{i}\right)+b+\varepsilon_{i}(\mathrm{i}=1,2, \ldots, 1)$

where $\varnothing(\cdot): \mathbb{R}^{p} \rightarrow \mathbb{R}^{n_{h}}$ is the mapping to a high dimensional (and possibly infinite dimensional) feature space, and the residuals e are assumed to be independent and identically distributed with zero mean and constant and finite variance.

\section{Proposed methods}

\subsection{Principle of least squares support vector machine regression}

Least squares support vector machine (LSSVM) formulates a regularized cost function and changes its inequation restriction to equation restriction. As a result, the solution process becomes a solution of a group of equations which greatly accelerates the solution speed [7]. To solve the problem stated in (1), the following optimization problem with a regularized cost function is formulated:

$$
\min _{w, b, \varepsilon_{i}}\left(\frac{1}{2} w^{T} w+\frac{C}{2} \sum_{i=1}^{l} \varepsilon_{i}^{2}\right)
$$

The solution of LS-SVM regressor will be obtained after we construct the Lagrangian function. The extreme point of $Q$ is a saddle point. Differentiating Q and using Lagrange multipliers, one obtains. The conditions for optimality are:

$\frac{\partial Q}{\partial w}=w-\sum_{i=1}^{l} \alpha_{i} \emptyset\left(x_{i}\right)=0$

$\frac{\partial Q}{\partial b}=-\sum_{i=1}^{l} \alpha_{i}=0$

$\frac{\partial Q}{\partial \alpha}=w^{T}-\varnothing\left(\mathrm{x}_{\mathrm{i}}\right)+\mathrm{b}+\varepsilon_{i}-y_{i}=0$

$\frac{\partial Q}{\partial \varepsilon_{i}}=C \varepsilon_{i}-\alpha_{i}=0$

where $\alpha \in \mathbb{R}$ are the Lagrange multipliers.

From formulas above, we can obtain:

$\frac{1}{2} \sum_{i=1}^{l} \alpha_{i} \varnothing\left(x_{i}\right) \sum_{j=1}^{l} \alpha_{j} \varnothing\left(x_{j}\right)+\frac{1}{2 C} \sum_{i=1}^{l} \alpha_{i}^{2}+$

$b \sum_{i=1}^{l} \alpha_{i}=\sum_{i=1}^{l} \alpha_{i} y_{i}$

The formula above can be expressed in matrix form:

$\left[\begin{array}{cc}0 & e^{T} \\ e & \Omega+C^{-1} I\end{array}\right](l+1)(l+1)\left[\begin{array}{l}\mathrm{b} \\ \alpha\end{array}\right]=\left[\begin{array}{l}0 \\ \mathrm{Y}\end{array}\right]$

In this equation,

$$
\begin{gathered}
e=[1, \ldots, 1]_{x}^{T} \\
\Omega_{i j}=K\left(x_{i}, x_{j}\right)=\varnothing\left(x_{i}\right)^{T} \emptyset\left(x_{j}\right)
\end{gathered}
$$

Formula (7) is a linear equation set corresponding to the optimization problem and can provide us with $\alpha$ and $b$. Thus, the prediction output decision function is:

$\bar{y}(x)=\sum_{i=1}^{l} \alpha_{i} K\left(x_{i} x\right)+b$

where $K\left(x_{i}, x\right)$ is the core function.

\subsection{Fundamentals of neural networks with levenberg-marquardt optimization}

The proposed neural network model is a two-layer feedforward network, with a sigmoid transfer function in the hidden layer and a linear transfer function in the output layer, as shown in Fig. $1 . \mathrm{W}$ is the weight matrix, and $\mathrm{b}$ is the bias. 


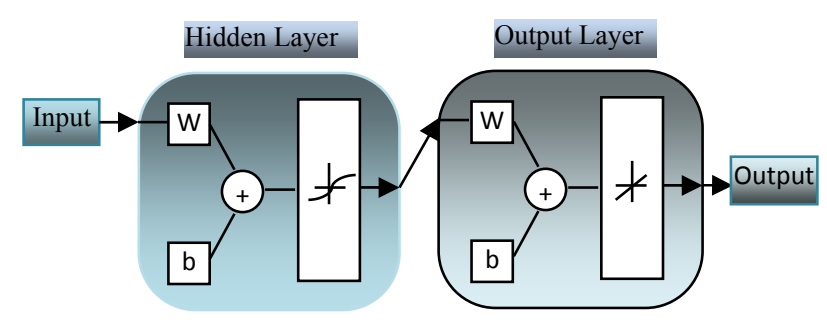

Fig. 1. Neural networks architecture for the predictive model. The network is a two-layer feedforward network, with a sigmoid transfer function in the hidden layer and a linear transfer function in the output layer. This network also uses tapped delay lines to store previous values of $y(t)$ sequences. W is the weight matrix, and $b$ is the bias.

In this section, the above neural network is trained using Levenberg-Marquardt backpropagation algorithm. It is a network training function that updates weight and bias values according to Levenberg-Marquardt optimization. It is often the fastest backpropagation algorithm for training moderate-sized feedforward neural networks (up to several hundred weights), although it does require more memory than other algorithms, such as conjugate gradient backpropagation.

Like the quasi-Newton methods, the Levenberg-Marquardt algorithm was designed to approach second-order training speed without having to compute the Hessian matrix. When the performance function has the form of a sum of squares (as is typical in training feedforward networks), then the Hessian matrix can be approximated as

$H=J^{T} J$

and the gradient can be computed as

$g=J^{T} e$

where $\mathrm{J}$ is the Jacobian matrix that contains first derivatives of the network errors with respect to the weights and biases, and $\mathrm{e}$ is a vector of network errors. The Jacobian matrix can be computed through a standard backpropagation technique that is much less complex than computing the Hessian matrix.

The Levenberg-Marquardt algorithm uses this approximation to the Hessian matrix in the following Newton-like update [8]:

$x_{k+1}=x_{k}-\left[J^{T} J+\mu I\right]^{-1} J^{T} e$

When the scalar $\mu$ is zero, this is just Newton's method, using the approximate Hessian matrix. When $\mu$ is large, this becomes gradient descent with a small step size. Newton's method is faster and more accurate near an error minimum, so the aim is to shift toward Newton's method as quickly as possible. Thus, $\mu$ is decreased after each successful step (reduction in performance function) and is increased only when a tentative step would increase the performance function. In this way, the performance function is always reduced at each iteration of the algorithm.

\section{Experimental results and discussion}

For experimental study, AISI 4340 steel and AISI D2 steel are used as the workpiece materials with average surface hardness values of 50, 55, and $60 \mathrm{HRC}$, respectively [9]. The cutting parameters include cutting speed $(\mathrm{m} / \mathrm{min})$, feed rates $(\mathrm{mm} / \mathrm{rev})$, and depths of cut $(\mathrm{mm})$. The data set has a total of 34 combinations of the cutting parameters with the actual surface roughness.

First we performed a normal probability plot on the residuals to identify substantive departures from normality, as shown in Fig. 2. The normal probability plot of the residuals appears as a straight line, which indicates that the assumption of normal distribution is valid [10].

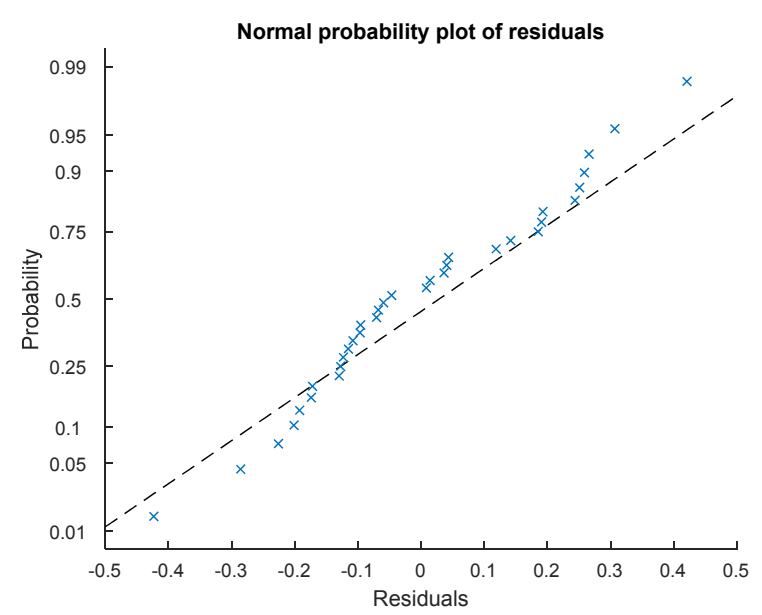

Fig.2. Normal probability plot of residuals.

The plot of the residuals versus the fitted values is illustrated in Fig. 3. Given that the dots are evenly distributed around the abscissa without a clear trend, the errors are independently distributed and the variance is constant [11]. 


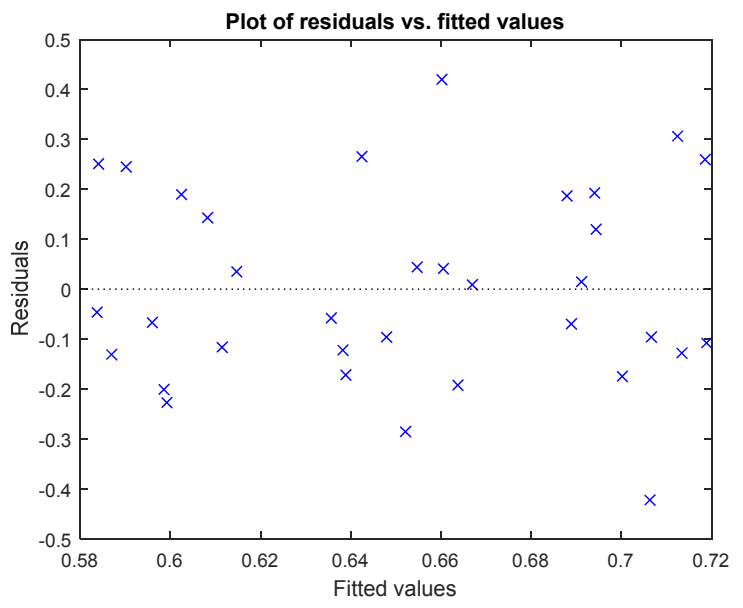

Fig.3. Plot of residuals versus fitted values.

\subsection{Experimental results for neural networks}

The mean squared errors (MSE) for the training, validation, and testing results are shown in Fig. 4. Each input contains the three cutting parameters, i.e. cutting speed $(\mathrm{m} / \mathrm{min})$, feed rates $(\mathrm{mm} / \mathrm{rev})$, and depths of cut $(\mathrm{mm})$. The surface roughness will be used as the target. In this research work, the first 24 input (i.e. $70 \%$ ) will be used as training data, 5 input (i.e. $15 \%$ ) will be used as validation data, and the remaining 5 input (i.e. 15\%) will be used as testing data. Training automatically stops when generalization stops improving, as indicated by an increase in the mean square error of the validation samples.

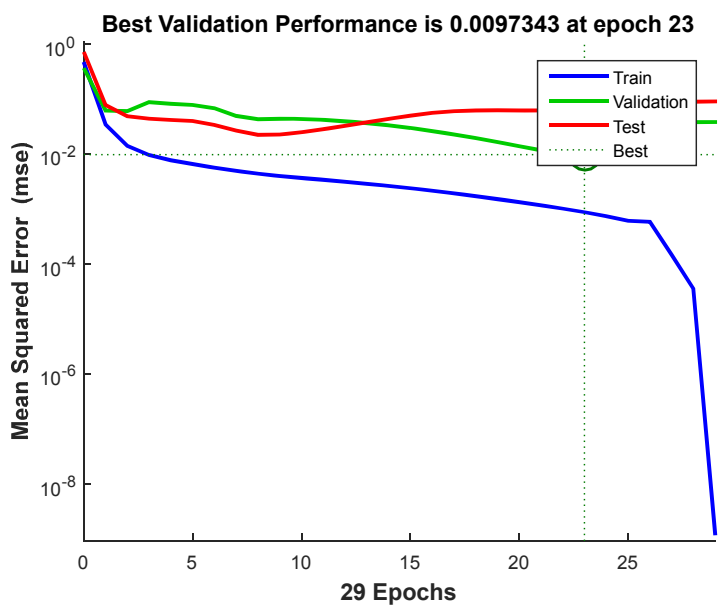

Fig.4. MSE for the training, validation, and testing data.
The best number of hidden neurons has determined to be 10. According to Fig. 4, the best validation error is as low as 0.0097343 at epoch 23. The training error is $9.08888 \mathrm{e}-4$ and the testing error is $1.09510 \mathrm{e}-1$ accordingly.

It is worthwhile studying the linear regression between the predicted surface roughness and the desired surface roughness. The correlation plot is shown in Fig. 5. The $\mathrm{x}-$ axis represents the target, and the y-axis represents the predicted surface roughness. The dashed line represents the perfect result. i.e. outputs $=$ targets. The solid line represents the best fit linear regression line between outputs and targets. The fit is reasonably good for the testing data, with $\mathrm{R}$ value of 0 . 93638. In addition, the linear regression line has an equation of the form Output $\sim=0.41 *$ Target + 0.2 .

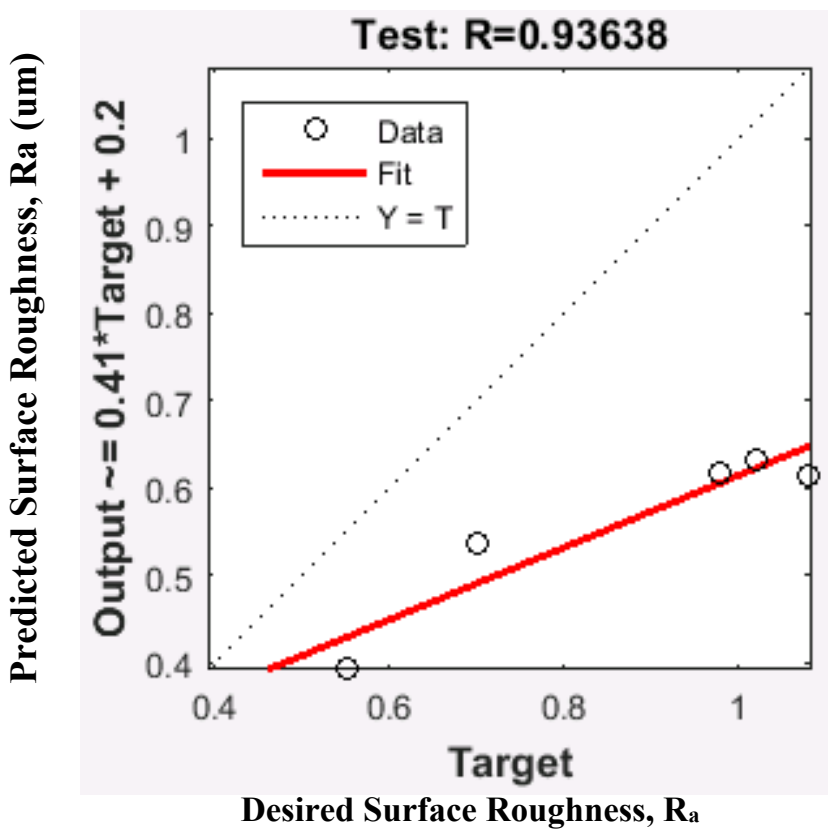

Fig.5. The regression plot between the predicted surface roughness, i.e. output and the desired surface roughness, i.e. target. The $\mathrm{x}$-axis represents the target, and the y-axis represents the predicted surface roughness. Five samples were used as the testing data.

We also investigated the corresponding change of gradient and $\mu$ in (13), which play an important role in reducing the mean squared errors faster and more accurate. As shown in Fig. 6, the gradient continuously decreases and reaches 0.00094178 at epoch 8 . Notably, $\mu$ is decreased to zero after one epoch and remains zero. This indicated that it took only one epoch for the training scheme to become Newton's 
method, which leads to a faster and more accurate near an error minimum. The validation check increases to 6 at epoch 8 .

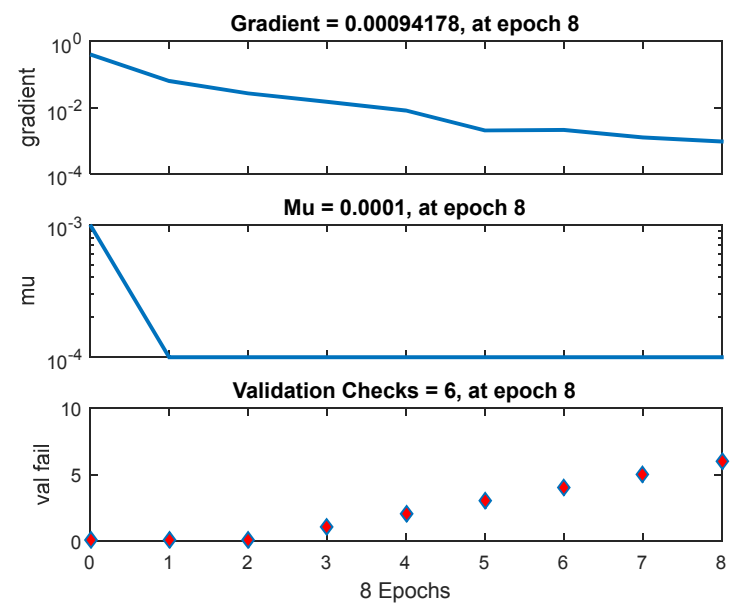

Fig.6. Observations of gradient, $\mu$, and validation checks.

4.2. Least Squares Support Vector Machine (LS-SVM)

The training process of LS-SVM involves the selection of kernel parameter: the squared bandwidth, $\sigma^{2}$ and the regularization constant, $\gamma$. $\gamma$ determines the trade-off between the training error minimization and smoothness. A good choice of these parameters is crucial for the performance of the estimator.

In this paper, the tuning parameters were found by using a state-of-the-art global optimization technique, Coupled Simulated Annealing (CSA) [12]. It determines suitable parameters according to some criterion and then passes these parameters to a multidimensional unconstrained non-linear optimization method, simplex to perform a fine-tuning step. Simplex finds a local minimum of a function starting from an initial point $X$. The local minimum is located via the Nelder-Mead simplex algorithm [13].

We used 10-fold cross-validation for selecting these parameters. The Kernel type is chosen to be RBF kernel. It is based upon feedforward simulation on the validation set using the feedforwardly trained model [14]. It breaks data into 10 sets of size of $n / 10$ if we assume the size of the data is $n$, then train on 9 datasets and test on 1 , and then repeat 10 times and then take a mean accuracy. The mean absolute error is used in combination with the 10 -fold cross-validation method [15]. It is the absolute value of the difference between the forecasted value and the actual value.

Another important choice is the selection of regressors. This selection is done by using a large number of initial components and then performing a greedy search to prune noninformative lags on a cross-validation basis [16]. Therefore an initial model containing all regressors is estimated and optimal choices for the parameters are made. On each stage of the greedy backwards elimination process, a regressor is removed if the cross-validation mean absolute error or mean squared error improves. For the purpose of model estimation, all series are normalized to zero mean and unit variance. Once the parameters are calculated, the final set of regressors is then used for the predictions.

The parameter tuning results are shown in Table 1. After 12 iterations, the $\gamma$ and $\sigma^{2}$ are optimized to the best values, 1.8392 and 0.099676 , respectively.

Table 1

Tuning of regularization constant, $\gamma$ and the kernel parameter, $\sigma^{2}$.

\begin{tabular}{|c|c|c|c|}
\hline Iteration & Minimum f(x) & $\mathbf{L n}(\gamma)$ & $\operatorname{Ln}\left(\boldsymbol{\sigma}^{2}\right)$ \\
\hline 1 & $1.153013 \mathrm{e}-02$ & 1.9712 & -1.4594 \\
\hline 2 & $1.153013 \mathrm{e}-02$ & 1.9712 & -1.4594 \\
\hline 3 & $1.151618 \mathrm{e}-02$ & 2.4212 & -1.7594 \\
\hline 4 & $1.151618 \mathrm{e}-02$ & 2.4212 & -1.7594 \\
\hline 5 & $1.151618 \mathrm{e}-02$ & 2.4212 & -1.7594 \\
\hline 6 & $1.151008 \mathrm{e}-02$ & 2.0134 & -2.0501 \\
\hline 7 & $1.149605 \mathrm{e}-02$ & 1.4532 & -2.0079 \\
\hline 8 & $1.149004 \mathrm{e}-02$ & 1.0454 & -2.2985 \\
\hline 9 & $1.149004 \mathrm{e}-02$ & 1.0454 & -2.2985 \\
\hline 10 & $1.148945 \mathrm{e}-02$ & 0.4214 & -2.4122 \\
\hline 11 & $1.148945 \mathrm{e}-02$ & 0.4214 & -2.4122 \\
\hline 12 & $1.148706 \mathrm{e}-02$ & 0.6093 & -2.3058 \\
\hline
\end{tabular}

\section{Comparisons of evaluation results}

A comparative study was performed to compare the experimental results among ANOVA, neural networks (NN), and LS-SVM. The considered performance criteria were the mean squared error (MSE), average relative error (ARE) and the determination coefficient [17] as follows:

$M S E=\frac{1}{N} \sum_{i=1}^{N}\left(y_{i}-\widehat{y}_{l}\right)^{2}$ 
$A R E=\frac{1}{N} \sum_{i=1}^{N}\left|\frac{y_{i}-\widehat{y_{l}}}{y_{i}}\right|$

$D_{c}^{2}=1-\left(\frac{\sum_{i}\left(y_{i}-\widehat{y}_{l}\right)^{2}}{\sum_{i} \widehat{y}_{l}^{2}}\right)$

where $\mathrm{N}$ is the number of patterns, and $y_{i}$ and $\widehat{y}_{l}$ denote the measured and predicted values, respectively.

The comparison of ANOVA, NN, and LSSVM on the surface roughness prediction was conducted. The MSE, ARE, and $D_{c}^{2}$ for ANOVA, NN, LS-SVM, and actual surface roughness are illustrated in Fig. 7.

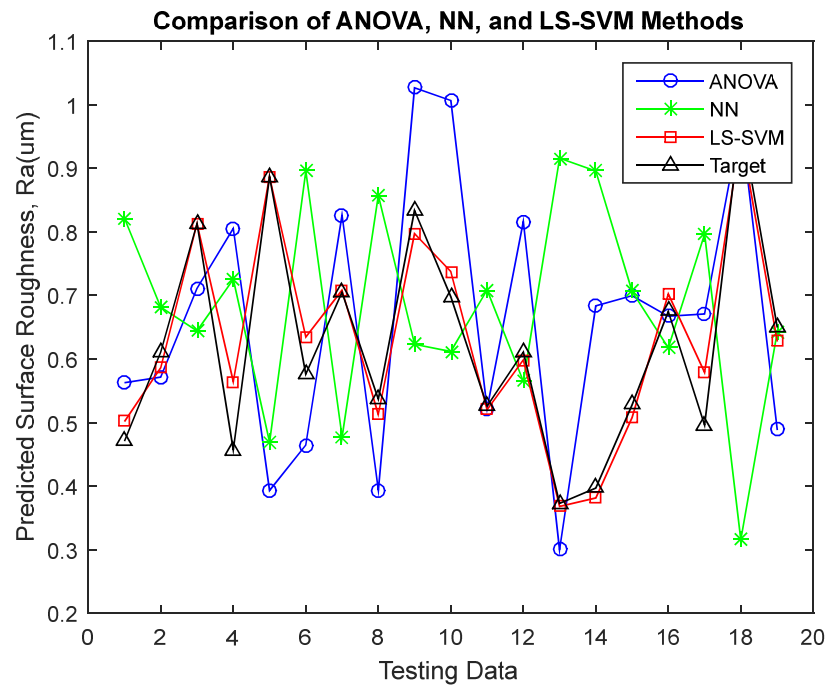

Fig.7. Comparison of ANOVA, neural networks, and LS-SVM methods. While the first 15 inputs in the data set were used as training data, the last 19 inputs were used as the testing data. ANOVA is displayed in blue circles, NN is represented in green stars, LS-SVM is illustrated in red square, and the target is given in black triangles.

To quantify these estimation errors, the MSE, ARE, and $D_{c}^{2}$ for ANOVA, NN, and LSSVM are summarized in Table 2. Compared with ANOVA, NN methods, the LS-SVM model produced the best results in terms of MSE, ARE, and $D_{c}^{2}$.

Table 2

Comparison of MSE, ARE, and $\mathbf{D}_{\mathbf{c}}^{2}$.

\begin{tabular}{|c|c|c|c|}
\hline Models & MSE & ARE & $\boldsymbol{D}_{\boldsymbol{c}}^{2}$ \\
\hline ANOVA & 0.2028 & 0.2674 & 0.1917 \\
\hline NN & 0.3123 & 0.4242 & 0.7266 \\
\hline LS-SVM & 0.0395 & 0.0477 & 0.9439 \\
\hline
\end{tabular}

\section{Conclusions}

In this paper, an effective least squares support vector machine (LS-SVM) based algorithm was developed to predict the surface roughness in machined surface. The real AISI4340 steel and AISID2 steel data set was used to conduct the experiments. We built an LS-SVM model by tuning the regularization constant, $\sigma^{2}$ and the kernel parameter, $\gamma$. A Gaussian Radial Basis Function (RBF) kernel framework was built on the data set to optimize the tuning parameters. The 10-fold crossvalidation method was used to estimate the generalization performance of the model. Then we trained the support values and the bias term of the LS-SVM for function approximation. We developed an effective training scheme for the model. After the network has been well trained, we tested both the training and prediction performance by predicting surface roughness on the training and test samples.

The promising experimental results demonstrated that the proposed LS-SVM based predictive model has superior prediction performance to the analysis of variance (ANOVA) method and the neural networks model trained by Levenberg-Marquardt algorithm. Specifically, the proposed LS-SVM algorithm produced a determination coefficient of $D_{c}^{2}=0.9439$, which is higher than the ANOVA and NN results of 0.1917 and 0.7266 . In addition, the parameter tuning method and the training scheme worked effectively, which ensure an accurate prediction of surface roughness.

\section{Acknowledgement}

This work was supported in part by the U.S. National Science Foundation under Grants HRD \#1505509 and HRD \#1533479, and USGS Grant under DC WRRI Grant \#2015DC174B.

\section{References}

[1] S. Moslehpour, C. Campana, D. Shetty, B. Deryniosky, Stand-alone surface roughness analyzer, IEEE Transactions on 
Instrumentation and Measurement, 58 (3), (2009) 698-706.

[2] D. Shetty, H. Neault, Method and apparatus for surface roughness measurement using laser diffraction pattern, United States Patent, Patent Number: 5,189,490 (1993).

[3] K. Sonar, U.S. Dixit, D.K. Ojha, The application of a radial basis function neural network for predicting the surface roughness in a turning process," International Journal Advanced Manufacturing Technology, 27 (2006) 661-666.

[4] K. M. Naidu, S. R. Rao, ANN based surface roughness prediction in turning of AA 6351, International Journal of Engineering Research and Applications (IJERA), 3 (4) (2013) 1455-1459.

[5] U. Caydas, S. Ekici, Support vector machines models for surface roughness prediction in CNC turning of AISI 304 austenitic stainless steel, Journal of Intelligent Manufacturing, 23 (3) (2012) 639-650.

[6] P. Wang, Q. Meng, J. Zhao, J. Li, X. Wang, Prediction of machine tool condition using support vector machine, Journal of Physics Conference, 1 (2011).

[7] J.A.K. Suykens, J. De Brabanter, L. Lukas, J. Vandewalle, "Weighted Least Squares Support Vector Machines: Robustness and Sparse Approximation," Neurocomputing, vol. 48, no. 1-4, pp. 85-105, 2002.

[8] M. Gan, H.X. Li, An Efficient Variable Projection Formulation for Separable Nonlinear Least Squares Problems, IEEE Transactions on Cybernetics, 5 (44) (2015) 707-711.

[9] K. He, Q, Xu, Q., M. Jia, "Modeling and Predicting Surface Roughness in Hard Turning Using a Bayesian Inference-Based HMM-SVM Model," IEEE Transactions on Automation Science and Engineering, vol. PP, no.99, pp.1-12, 2014.

[10] K. Hinkelmann, Design and Analysis of Experiments, Special Designs and Applications. Hoboken, NJ, USA: Wiley, (2012).

[11] R. L. Scheaffer, M. S. Mulekar, and J. T. McClave, Probability and satistics for engineers, 5th ed. Boston, MA, USA: Cengage Learning (2010).

[12] S. Xavier-de-Souza, J.A.K. Suykens, J. Vandewalle, D. Bolle, Coupled Simulated Annealing, IEEE Transactions on Systems, Man and Cybernetics - Part B, 40 (2), (2010) 320-335.

[13] J. A. Nelder, R. Mead, A simplex method for function minimization," Computer Journal, 7 (1965) 308-313.

[14] N. Zhang, T. Alemayehu, P. Behera, Nonlinear autoregressive (NAR) forecasting model for potomac river stage using least squares support vector machines (ls-svm)", International Journal of Innovative Technology and Exploring Engineering (IJITEE), 4 (9) (2015) 1-9.

[15] N. Zhang, R. Kamaha, P. Behera, Prediction of surface water supply sources for the district of columbia using least squares support vector machines (ls-svm) method, Advances in Computer Science: an International Journal, 4 (1) (2014) 47-51.

[16] N. Zhang, C. Williams, P. Behera, Water quantity prediction using least squares support vector machines (1s-svm) method, Journal on Systemics, Cybernetics and Informatics (JSCI), 12 (4) (2014) 53-58.

[17] î. Asiltürk, M. Çunkas, Modeling and prediction of surface roughness in turning operations using artificial neural network and multiple regression method, Expert Syst. Appl., 38 (5), (2011) 5826-5832.

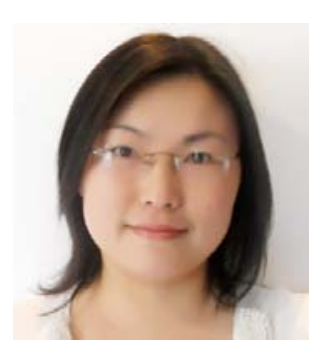

Dr. Nian Zhang received her B.S. degree (with honor) in Electrical Engineering from Wuhan University of Technology, M.S. degree in Electrical Engineering from Huazhong University of Science and Technology, and Ph.D. degree in Computer Engineering from Missouri University of Science and Technology. She is an Assistant Professor of the Department of Electrical and Computer Engineering at the University of the District of Columbia, Washington D.C. Dr. Zhang's research expertise and interests include computational intelligence, machine learning and data mining, and various application fields including big data science, pattern recognition, time series prediction, biomedical applications, and autonomous robot navigation. Her 
research has been funded by National Science Foundation (NSF), Office of Naval Research (ONR), NASA, US Geological Survey (USGS), Xerox Corporation, and Bush Foundation. Dr. Zhang is an ONR Summer Faculty Research Fellow and IEEE Senior Member. She is an Associate editor of the IEEE Transactions on Neural Networks and Learning Systems, and Guest Editor of Computational Intelligence and Neuroscience Journal and International Journal of Systems, Control and Communications. Dr. Zhang was a recipient of the Best Paper Award in the 2003 IEEE International Conference on Fuzzy Systems (FUZZ-IEEE).

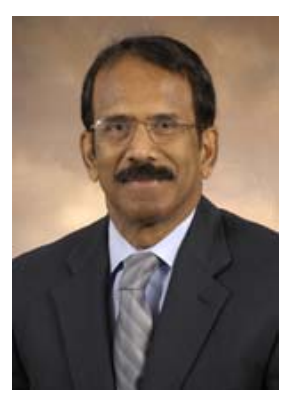

Dr. Devdas Shetty is the Dean of School of Engineering and Applied Sciences at University of the District of Columbia since 2012, having previously served as Dean of Engineering at Lawrence Technological Institute and Dean of Research at the University of Hartford. While with the University of Hartford, Dr. Shetty was first Chair of the Vernon D. Roosa Endowed Professorship. In addition, he was the Director of the Engineering Applications Center, through which he established partnerships with more than 50 Connecticut industries. During 2008 and 2009, Dr. Shetty served as Dean of the College of Engineering for Lawrence Technological University in Michigan . During that time, he initiated several new academic programs, established partnerships and contributed to curricular innovation. Prior to coming to Hartford, Dr. Shetty held academic positions at the Albert Nerkin School of Engineering at the Cooper Union for the Advancement of Science and Art in New York City. Dr. Shetty is the author of three books and more than 200 scientific articles and six patents. His books on Mechatronics and Product Design are widely used as a textbooks in many universities around the world. Dr. Shetty's research work has been cited for original contribution to the understanding of engineering surface measurement, for significant intellectual achievements in mechatronics and for contributions to product design. He is especially well-known for his contributions in establishing partnerships between the University and industries. He is the recipient of academic and research grants from organizations like National Science Foundation, Society of Manufacturing Engineers, US Army, Air force etc. Dr. Shetty had been leading research efforts in a U.S. Army research project on Unmanned Aerial Vehicles. In partnership with Albert Einstein College of Medicine in New York, he invented the patented mechatronics process for supporting patients. Dr. Shetty has chaired several international conferences and presented keynote lectures. Major honors received by Prof. Shetty include James Frances Bent award for Creativity, the Edward S. Roth National Award for Manufacturing from the Society of Manufacturing
Engineers, American Society of Mechanical Engineer Faculty Award, and Society of Manufacturing Engineers Honor award. He is an elected member of the Connecticut Academy of Science and Engineering. 